\title{
Dynamic spin-current generation in hybrid structures by sound wave
}

\author{
M.S. Okorokov ${ }^{1}$, I.I. Lyapilin ${ }^{1,2}$, and V.V. Ustinov ${ }^{1}$ \\ ${ }^{1}$ M.N. Mikheev Institute of Metal Physics, UB RAS, Ekaterinburg 620990, Russia \\ E-mail: Okorokovmike@gmail.com \\ ${ }^{2}$ Ural Federal University, Ekaterinburg 620990, Russia
}

Received December 6, 2016, published online February 24, 2017

\begin{abstract}
We have developed the theory of spin transport transverse to the interface in metal/ferromagnetic insulator hybrid structures under the spin Seebeck effect conditions. We have calculated the deviation of the conduction electrons spin temperature from equilibrium under conditions of saturation of resonance interaction between the electrons and a sound wave field. We have demonstrated that the conduction electrons spin subsystem, when excited under the above conditions, generates a spin-wave current in a nonconducting ferromagnetic of the metal/ferromagnetic insulator hybrid structure being in a nonuniform temperature field. In addition, the spin-wave current generation in the ferromagnetic has a resonance nature. We have analyzed the approximation of effective parameters, when each of the considered subsystems (conduction electrons, magnons, and phonons) is characterized by its effective temperature.
\end{abstract}

PACS: $43.20 . F n$ Scattering of acoustic waves;

43.20.Ks Standing waves, resonance, normal modes;

43.20. $\mathrm{Tb}$ Interaction of vibrating structures with surrounding medium;

43.25.Gf Standing waves; resonance;

43.35.Xd Nuclear acoustical resonance, acoustical magnetic resonance.

Keywords: spin-wave current, sound wave, resonance, phonon, thermoelectric coefficients.

\section{Introduction}

Thermoelectric and thermomagnetic effects reflect the relationship between charge and heat currents, and as turned out, may cause spin effects. The spin Seebeck effect (SSE) observed in $\mathrm{Ni}_{81} \mathrm{Fe}_{19}$ crystals was the first step to open a new direction in spintronics - studying the influence of thermal perturbations on spin effects [1,2]. Afterwards, the SSE could be observed in various materials, both semiconductors (Ga,Mn)As [2] and metallic ferromagnets $\mathrm{Co}_{2} \mathrm{MnSi}$ [3]. Later, other spin effects have been discovered: the Nerst effect, the spin Peltier effect, etc. [4-9]. Thus, spintronics that investigates charge- and heat-current interaction initiates a new field of research in physics - spin caloritronics [10,11]. Spin effects in isolating magnetic materials under the influence of thermal perturbations, were studied in [12-15].

The SSE's investigation results proved to be unexpected in the context of a nonmagnetic conductor $\mathrm{LaY}_{2} \mathrm{Fe}_{5} \mathrm{O}_{12}$ magnetic insulator system $[16,17]$. The SSE measurements have been taken using the inverse spin Hall effect (ISHE).
Against conducting crystals where the transfer of the spin angular momentum is due to band charge carriers, in nonconducting magnetic materials the spin Seebeck effect can be realized by exciting a localized spin system. For SSE, the angular momentum transfer is driven by a spin-wave current (spin wave) underlain by excitations of the localized spin subsystem (magnons). Since spin waves relax weakly enough, the spin-wave current propagates far greater distances than the electron spin current. This circumstance promises possible practical applications of the effect $[18,19]$. Being in thermodynamic equilibrium, the magnons are described by the Bose-Einstein distribution function. A nonequilibrium magnon distribution induced by a thermal gradient causes the momenta to distribute chaotically and never provides the spin-wave current. The spin-wave current excitation requires producing a nonequilibrium but "coherent" in momentum direction magnon distribution. Such a distribution can be formed, for example, as a result of inelastic electron scattering by localized magnetic impurity centers nearby the structure interface. Let the spin-polarized distribution of conduction electrons in a nonmagnetic metal (the spin 
accumulation) be obtained in that or other way. Then, the magnon creation process dominates the annihilation process as the localized magnetic momenta scatter the electrons inelastically. In this case, the magnon distribution generates the spin-wave current. As a rule, the spin Hall effect is used to give rise to the spin accumulation [20]; its effectiveness depends on the magnitude of the spin-orbital interaction in a conduction electron system. That is why platinum whose spin-orbital interaction is high enough needs to apply.

Spin currents are quite difficult to detect or generate. At first, detections of spin currents have been carried out by indirect methods, by measuring the effects accompanied with the spin current generation. So, the work [21] uses optical methods to measure the spin accumulation occurring on the lateral surfaces of the sample when generating the spin current in spin-Hall systems. Subsequently, the inverse spin Hall effect has been proposed as an electrical method of detecting the spin currents $[20,22,23]$. The essence of this method is based on inducing a voltage by the spin current against the background of the spin-orbital interaction $[18,24]$. For the first time such a spin current detection method has been demonstrated in the work [25] and has actually become the main method of detecting spin currents [26-28]. Searching and development of new methods of spin-current generation are of current interest.

Among other methods which can bring the conduction electron subsystem to the spin-polarized state, resonant ones should be mentioned. Resonant methods implementation for generating electron spin current in magnetic insulator nonmagnetic metal structures was studied in [26,29]. The method is about resonant excitation of localized moments system by alternating external fields of different nature and an electron spin current generation in the nonmagnetic metal attached to the magnetic insulator. This way provides the spin current without transferring spin-polarized charge carriers through the interface in the hybrid structures. As such, the approach avoids the mismatch problem [30-33] that prevents to obtain high spin polarization values by injection of spin-polarized electrons. Electron spin current excitation under acoustic resonance conditions was studied in $[34,35]$. It is of interest to study the implementation of the resonant method for spin accumulation creation under SSE conditions in metal/magnetic insulator/metal structures. Such a resonant scenario can be possible to implement in the event of taking the spin-orbital interaction (SOI) into account. SOI couples the kinetic (translational) and spin subsystems of conduction electrons, therefore, it can be regarded as one of the possible channels to act on one of the subsystems via another, for example, on the spin subsystem of conduction electrons via the kinetic subsystem and vice versa. Due to the translational and spin motion locking, the quantum transitions cannot be conventionally divided into pure configurational (orbital) and pure spin ones. We can talk only about either predominantly configurational or predominantly spin transitions. But this circumstance significantly changes the conditions for the excitation of different transitions. Namely, the electrical component of an electromagnetic field initiates the spin transitions, and the magnetic component - the orbital ones. The spin-orbit interaction gives rise to resonant electron transitions at frequencies being linear combinations of the cyclotron and Zeeman frequencies. Besides, such transitions can exist at the antinode of both electric and magnetic fields. Such resonance is known as the combined Rashba resonance [36-39] and observed in a number of compounds.

There are other possible perturbations causing the resonance, for example, the interaction between conduction electrons and a sound wave field. The sound wave propagation in a crystal is accompanied with excitation of an electromagnetic field with potentials $\varphi(\mathbf{x}, t)$ and $\mathbf{A}(\mathbf{x}, t)$ and corresponding strengths of the electric and magnetic fields. In this case, the kp model Hamiltonian of the perturbation theory for a crystal in the sound wave field, in general, contains a number of summands: the interaction of electrons with the scalar potential and an alternating magnetic field of the accompanying electromagnetic wave; the spin-orbital interaction of electrons with an electric field $\mathbf{E}$ that accompanies the sound wave [40]; the spin-independent deformational interaction of electrons with the displacement field [41]; the spin-dependent deformational interaction [40], etc. Along with this, there are a few spin interaction mechanisms when the sound wave or the corresponding electromagnetic wave modulates the interaction between kinetic and spin degrees of freedom. The mechanisms listed above differ from each other not in the interaction intensity but in the line widths and resonant frequency positions. The works $[42,43]$ demonstrates that the interaction between conduction electrons and a sound wave field also causes a spin current. It should be here noted that the paper [44] shows experimentally that the response of the spin electron subsystem (analogous to the spin Hall effect), when interacted with the sound wave field, has a resonant nature.

It can be said that the basic mechanisms involved in the spin absorption of ultra-sound by free electrons in crystals are: the modulation of the dipole-dipole interactions of electron spins by sound [45]; the interaction of electron spins with an alternating magnetic field accompanying the sound wave [45]; the sound modulation of the interaction between spin and kinetic degree of freedom of conduction electrons in crystals without inversion centers [36]; the sound modulation of the spin-orbital interaction between electrons and a crystal lattice [46,47]. The mechanisms mentioned above differ from each other not in the interaction intensity but in the line widths and resonant frequency positions. In general case, the interaction of conduction electrons with sound has a resonant nature. The resonance occurs when the sound frequency $\omega$ and the spin precession frequency $\omega_{s}$ coincide. A similar picture can be observed at other frequencies being a linear combination of the Zeeman $\omega_{s}$ and cyclotron $\omega_{c}$ frequencies. Against the paramagnetic resonance (PR), the acoustic spin reso- 
nance (ASR) can be observed both in the longitudinal and in the transverse polarization of sound waves.

Thus, taking SOI into account leads to resonance influence on electron spin subsystem, without affecting localized spin subsystem. It is also result in electron spin polarization which is nessesary for realization SSE in resonance saturation conditions in such kind of structures. Correct description of the thermal perturbation is the other important question in the thermal effects analyzing. There are few possible ways for taking such perturbations into account. The response of a weakly nonequilibrium system on a thermal type perturbation can be represented as a Fourier transforms of the time correlation functions of the operators for corresponding flows with statistically equilibrium system state [48]. The admittances of this type structure is similar to the expressions for kinetic coefficients, emerging in the equilibrium systems theory as a reaction on mechanical type perturbation, which are representable in the form of an additional summand in the Hamiltonian of the system. Sometimes the reaction on thermal perturbations is found using indirect methods by putting in fictitious external forces acting on the system similarly to thermal perturbations [49]. The response to a thermal perturbation can be calculated using Onsager hypothesis [50] on the nature of the decay of fluctuations or using local equilibrium distribution as an initial condition for the finding of a statistical operator of the system [51]. Universal description of the response of weakly nonequilibrium systems on thermal type perturbations gives the nonequilibrium statistical operator method (NSO) [52]. The method allows us to calculate the response to an external perturbation not only of the equilibrium, but also of strongly nonequilibrium systems.

In the present work, the influence of resonant interaction of conduction electrons with the sound wave field on spinwave current generation in ferromagnetic part of the met$\mathrm{al} /$ ferromagnetic insulator system is described using NSO method for thermal perturbations describtion. The paper is organized as follows. In the first part the model of interest is formulated, the Hamiltonian of the system is described and the basic operators with its dynamics equations are introduced. Exchange interaction is considered to be the only mechanism responsible for electron spins relaxation on the localized spins near the interface. Magnon-phonon interaction is considered to be an additional way for magnons to scatter. The construction of both nonequilibrium entropy operator takes into account the thermal perturbations of the system and NSO is included to the second part of the work. The third part contains the analysis of macroscopic equations describing spin-thermal effects.

\section{Hamiltonian}

In our case, the problem of the spin-wave generation to be solved reduces to building and analyzing a set of macroscopic equations for two interacting spin subsystems: the conduction electron subsystem $\left\langle\dot{s}^{z}(\mathbf{x})\right\rangle^{t}$ and the subsystem of localized spins of an insulator $\left\langle\dot{S}^{z}(\mathbf{x})\right\rangle^{t}$. Here

$$
\begin{gathered}
\langle A\rangle^{t}=\operatorname{Sp}(\rho(t) A), \quad \dot{A}=(i \hbar)^{-1}[A, H], \\
A(\mathbf{x})=\sum_{i}\left\{A_{i}, \delta\left(\mathbf{x}-\mathbf{x}_{i}\right)\right\}, \quad\{A, B\}=(A B+B A) / 2,
\end{gathered}
$$

where $\rho(t)$ is the nonequilibrium statistical operator (or the density matrix), $H$ is the Hamiltonian of the system considered,

$$
H=H_{e}+H_{s F}+H_{m}+H_{p}+H_{s m}+H_{m p} .
$$

The system of conduction electrons in a normal metal is described by the Hamiltonian $H_{e}$

$$
\begin{gathered}
H_{e}=\int d x\left\{H_{k}(\mathbf{x})+H_{s}(\mathbf{x})\right\}, \quad H_{k}(\mathbf{x})=\sum_{j}\left\{\frac{p_{j}^{2}}{2 m}, \delta\left(\mathbf{x}-\mathbf{x}_{j}\right)\right\}, \\
H_{S}(\mathbf{x})=-\hbar \omega_{s} \sum_{j} s_{j}^{z} \delta\left(\mathbf{x}-\mathbf{x}_{j}\right) .
\end{gathered}
$$

Here $H_{k}(\mathbf{x}), H_{s}(\mathbf{x})$ are the density operators of kinetic and spin energies of electrons; $s_{j}^{z}$ and $p_{j}^{\gamma}$ are the spin and kinetic momentum operator components of the $j$ th electron, respectively; $\omega_{s}=g_{s} \mu_{0} H / \hbar$ is the Zeeman precession frequency of free electrons in an external magnetic field $\mathbf{H}=(0,0, H) ; g_{s}, \mu_{0}$ are the effective electron spectroscopic splitting factor and the Bohr magneton, respectively.

The spin interactions between electrons and a sound wave

$$
\mathbf{u}(\mathbf{x}, t)=\sum_{\mathbf{q}} \mathbf{u}(\mathbf{q}) \mathrm{e}^{i \mathbf{q} x+i \omega t}
$$

( $\omega=\mathbf{s q}, \mathbf{s}$ is a sound speed, $\mathbf{q}$ is a wave vector) can be represented as follows:

$$
H_{s F}(t)=\sum_{i n \mathbf{q}} \Phi_{-i}^{-n}(\mathbf{q}) u^{i}(\mathbf{q}) \mathrm{e}^{i \omega t} T^{n}(\mathbf{q}),
$$

where $\Phi_{-i}^{-n}(\mathbf{q})$ are matrices characterizing the interaction intensity. The tensor operators $T^{n}(\mathbf{q})$ depend on the group indices $n=\left(\mu, \alpha_{1}, \cdots\right)$ running values of $(+,-, 0)$ :

$$
T^{n}(\mathbf{q})=T(\mathbf{q})^{\mu, \alpha_{1}, \cdots}=\sum_{j}\left\{s_{j}^{\mu} p_{j}^{\alpha_{1}} \cdots, \mathrm{e}^{i q x_{j}}\right\} .
$$

The explicit form of the operator $T^{n}(\mathbf{q})$ is defined by a particular structure of the crystal. Each of the operators corresponds to a single certain line of the acoustic spin resonance. For definiteness, the operator $T^{n}(\mathbf{q})$ is thought to coincide with the Fourier-component of the spin distribution density [40]

$$
T^{n}(\mathbf{q})=\sum_{j}\left\{s_{j}^{\mu}, \mathrm{e}^{i q x_{j}}\right\}=s^{\mu}(\mathbf{q}) .
$$


Such a type of interaction between sound and spin degrees of freedom of conduction electrons can be realized in crystals of $\mathrm{Si}, \mathrm{Bi}, \mathrm{GdS}$, etc.

The Hamiltonian $H_{m}$ for the magnetic subsystem is of a sum of the exchange $H_{S S}(\mathbf{x})$ (over the nearest neighbors) and Zeeman energies $H_{S}(\mathbf{x})$ of the localized spins,

$$
\begin{gathered}
H_{m}=\int d \mathbf{x}\left\{H_{S S}(\mathbf{x})+H_{S}(\mathbf{x})\right\} \\
H_{S S}=-J \sum_{j \delta} S_{j} S_{j+\delta}, \quad H_{S}=-\hbar \omega_{m} \sum_{j} S_{j}^{z} .
\end{gathered}
$$

$J$ is the exchange integral, $\omega_{m}=g_{m} \mu_{0} H / \hbar$.

Going over to the creation $b_{v}^{+}$and annihilation $b_{v}$ magnon operators [54], we transform the Hamiltonian of the localized spin subsystem. Treating the magnon gas as free, we have

$$
H_{m}=\sum_{k} \varepsilon(k) b_{k}^{+} b_{k}, \text { where } \varepsilon(k)=\frac{\hbar^{2} k^{2}}{2 m^{*}} .
$$

The above expression can be interpreted as a sum of energies of the quasiparticles - magnons, having the quasimomentum $\mathbf{k}$ with its effective mass $m^{*}$ and magnon momentum [55].

The main mechanism of the spin scattering of the conduction electrons implies the exchange interaction with the localized spins located nearby the interface $H_{s m}$,

$$
H_{s m}=-J_{0} \sum_{j} \int d \mathbf{x s}(\mathbf{x}) \mathbf{S}\left(\mathbf{R}_{j}\right) \delta\left(\mathbf{x}-\mathbf{R}_{j}\right),
$$

$J_{0}$ is the exchange integral, $\mathbf{S}\left(\mathbf{R}_{j}\right)$ is the operator of a localized spin with the coordinate $\mathbf{R}_{j}$ at the interface, $H_{p m}(\mathbf{x})$ is the energy density operator of interaction between the localized spins and phonons.

$H_{p}$ is the lattice Hamiltonian

$$
H_{p}=\int d \mathbf{x} H_{p}(\mathbf{x})
$$

where $H_{p}(\mathbf{x})$ is the energy density operator for the phonon subsystem. The explicit form of which is not necessary for the following calculations.

We find an explicit expression for the statistical operator (or the density matrix) $\rho(t)$ using the nonequilibrium statistical operator method. By $[42,43,52,53]$, in the linear approximation in deviation from equilibrium $\rho(t)$ can be written as

$$
\begin{aligned}
& \rho(t)=\rho_{q}(t)-\int_{-\infty}^{0} d t_{1} \mathrm{e}^{\varepsilon t_{1}} \int_{0}^{1} d \tau \rho_{0}^{\tau} \dot{S}\left(t+t_{1}, t_{1}\right) \rho_{0}^{-\tau} \rho_{0}, \\
& \rho_{q}(t)=\exp \{-S(t)\}, \quad \rho_{0}=\exp \left\{-S_{0}\right\} .
\end{aligned}
$$

Here, $\rho_{q}(t)$ is the quasi-equilibrium statistical operator, $S_{0}=\Phi_{0}+\beta\left(H_{e}+H_{m}+H_{p}+H_{s m}+H_{m p}\right)$ is the entropy of the equilibrium system, $\beta^{-1}=T$ is the equilibrium temperature of the system; $\dot{S}(t)$ is the entropy production operator.

It should be noted that to implement resonant transitions at the combined frequencies, we should expect both the kinetic and spin subsystems of conduction electrons to be unbalanced. However, in the case of resonance at the spin frequency, only the conduction electron spin subsystem absorbs energy from the external field. In addition, the conduction electron kinetic subsystem appears to deviate but slightly from equilibrium. Therefore, under the spin resonance saturation conditions, it suffices to view the evolution of two spin subsystems: the conduction electron subsystem and the localized spin subsystem. The nonequilibrium state of our system can be then described by determining the average energies of the subsystems $(s),(m)$ or their conjugate inverse effective temperatures $\beta_{s}, \beta_{m}$. In other words, this fits the case of establishing the internal equilibrium inside each of the subsystems at a rate far greater than the energy exchange rate between them. The temperature $T_{p}=\beta_{p}^{-1}$ corresponds to the phonon subsystem. Earlier, we have exploited an analogous approach to describe the spin Hall effect in hybrid structures [42,43].

So, the entropy operator corresponding to the nonequilibrium state of the system can be written in terms of the average density values as follows:

$$
\begin{gathered}
S(t)=\Phi(t)+\Delta \int d \mathbf{x}\left\{\beta_{s}(\mathbf{x}, t)\left(H_{s}(\mathbf{x})+(1 / 2) H_{s m}(\mathbf{x})\right)+\right. \\
+\beta_{m}(\mathbf{x}, t)\left(H_{m}(\mathbf{x})+(1 / 2) H_{s m}(\mathbf{x})+(1 / 2) H_{p m}(\mathbf{x})\right)+ \\
\left.+\beta_{p}(\mathbf{x}, t)\left(H_{p}(\mathbf{x})+(1 / 2) H_{p m}(\mathbf{x})\right)\right\}=S_{0}+\delta S(t), \\
\Delta A=A-\langle A\rangle_{0}, \quad\langle\ldots\rangle_{0}=\operatorname{Sp}\left(\ldots \rho_{0}\right),
\end{gathered}
$$

$\Phi(t)$ is the Massieu-Plank functional.

\section{Microscopic equations}

Let us calculate the density of the electron spin current

$$
\begin{gathered}
\frac{d}{d t} s^{z}(\mathbf{x})=(i \hbar)^{-1}\left[s^{z}(\mathbf{x}), H\right]=\dot{s}_{(s m)}^{z}(\mathbf{x})+\dot{s}_{(s F)}^{z}(\mathbf{x}, t), \\
\dot{A}_{i k}(\mathbf{x})=(i \hbar)^{-1}\left[A(\mathbf{x}), H_{i k}\right] .
\end{gathered}
$$

The collisional expression $\dot{s}_{(s m)}^{z}(\mathbf{x})$ is governed by inelastic scattering of the electrons by the localized spins. These processes are accompanied by the creation and annihilation of magnons

$$
\dot{s}_{(s m)}^{z}(\mathbf{x})=\frac{i J_{0}}{\hbar} \sum_{i} \int d \mathbf{x}\left\{s^{+}(\mathbf{x}) S^{-}\left(\mathbf{R}_{i}\right)-s^{-}(\mathbf{x}) S^{+}\left(\mathbf{R}_{i}\right)\right\} \delta\left(\mathbf{x}-\mathbf{R}_{i}\right)
$$

The last summand on the right-hand side in (9) is responsible for the change in the spin current due to the electrons absorb energy of the sound wave. 
The equation of motion for the magnetic subsystem is

$$
\dot{H}_{m}(\mathbf{x})=-\nabla I_{H_{m}}(\mathbf{x})+\dot{H}_{m(s m)}(\mathbf{x})+\dot{H}_{m(p m)}(\mathbf{x}) .
$$

Here $I_{B}(\mathbf{x})=\sum_{i} B\left\{p_{i} / m, \delta\left(\mathbf{x}-\mathbf{x}_{i}\right)\right.$ is the flux density operator (for magnon energy in this case). The rest of the terms on the right-hand side of the equation are responsible for the interface-magnon and phonon-magnon scattering processes.

Ultimately, the equation of motion for the lattice subsystem has the form

$$
\dot{H}_{p}(\mathbf{x})=-\nabla I_{H_{p}}(\mathbf{x})+\dot{H}_{p(p m)}(\mathbf{x}) .
$$

Given the explicit form of the equations of motion, we can come to an expression for the entropy operator. Integrating by parts the terms containing the divergence of fluxes and discarding the surface integrals, we represent the entropy production operator as follows:

$$
\begin{gathered}
\dot{S}(t)=\Delta \int d \mathbf{x}\left\{I_{H_{m}}(\mathbf{x}) \nabla \beta_{m}(\mathbf{x}, t)+\right. \\
+I_{H_{p}}(\mathbf{x}) \nabla \beta_{p}(\mathbf{x}, t)+\beta \dot{H}_{s F}(\mathbf{x}, t)+ \\
+\beta_{p}(\mathbf{x}, t)(1 / 2) \dot{H}_{p(p m)}(\mathbf{x})+\beta_{s}(\mathbf{x}, t)(1 / 2) \dot{H}_{s(s m)}(\mathbf{x})+ \\
\left.+\beta_{m}(\mathbf{x}, t)(1 / 2)\left[\dot{H}_{m(s m)}(\mathbf{x})+\dot{H}_{m(p m)}(\mathbf{x})\right]\right\} .
\end{gathered}
$$

\section{Macroscopic equations}

At first, we insert the entropy production operator (13) into the expression for the nonequilibrium statistical operator (7). Then we average the operator equations (9), (11) using NSO and find macroscopic equations having the meaning of the local laws of conservation of the energy density of the subsystems $(s),(m)$. We get

$$
\begin{gathered}
\left\langle\dot{s}^{z}(\mathbf{x})\right\rangle^{t}=\left\langle\dot{s}_{(s m)}^{z}(\mathbf{x})\right\rangle^{t}+\left\langle\dot{s}_{(s F)}^{z}(\mathbf{x})\right\rangle^{t} \\
\left\langle\dot{S}^{z}(\mathbf{x})\right\rangle^{t}=-\nabla\left\langle I_{S^{z}}(\mathbf{x})\right\rangle^{t}+\left\langle\dot{S}_{(s m)}^{z}(\mathbf{x})\right\rangle^{t}+\left\langle\dot{S}_{(m p)}^{z}(\mathbf{x})\right\rangle^{t}
\end{gathered}
$$

Equations (14), (15) describes the change in density of the spin magnetization of the electronic and magnetic subsystems due to the following processes: diffusion (the first summand on the right-hand sides of the equation)

$$
\begin{aligned}
\left\langle I_{S^{z}}^{\alpha}(\mathbf{x})\right\rangle^{t} & =\int d \mathbf{x}^{\prime} \int_{-\infty}^{0} d t_{1} \mathrm{e}^{\varepsilon t_{1}}\left\{\left(I_{S^{z}}^{\alpha}(\mathbf{x}), I_{S^{z}}^{\lambda}\left(\mathbf{x}^{\prime}, t_{1}\right)\right) \nabla \beta_{m}^{\lambda}\left(\mathbf{x}^{\prime}, t+t_{1}\right)\right)+ \\
& \left.+\left(I_{S^{z}}^{\alpha}(\mathbf{x}), I_{H_{p}}^{\lambda}\left(\mathbf{x}^{\prime}, t_{1}\right)\right) \nabla \beta_{p}^{\lambda}\left(\mathbf{x}^{\prime}, t+t_{1}\right)\right\}
\end{aligned}
$$

Relaxation terms in the equations are

$$
\begin{aligned}
& \left\langle\dot{A}_{(j n)}(\mathbf{x})\right\rangle^{t}=\int d \mathbf{x}^{\prime} \int_{-\infty}^{0} d t_{1} \mathrm{e}^{\varepsilon t_{1}}\left(\dot{A}_{(j n)}(\mathbf{x}), \quad \dot{A}_{(j n)}\left(\mathbf{x}^{\prime}, t_{1}\right)\right) \times \\
& \quad \times \delta \beta_{j}\left(\mathbf{x}^{\prime}, t+t_{1}\right), \quad A=s^{z}, S^{z} ; j=s, m ; n=m, p .
\end{aligned}
$$

Here

$$
(A, B)=\int_{0}^{1} d \tau\langle\Delta A, \Delta B(i \hbar \beta \tau)\rangle_{0}, \quad B(t)=\mathrm{e}^{i t H / \hbar} B \mathrm{e}^{-i t H / \hbar} .
$$

The last term in the expressions for the spin-wave current (14) deals with the power absorbed by the conduction electrons as the sound wave propagates in the crystal. In accordance with [43], the average power $Q_{S}$, absorbed by the electrons is given by

$$
Q_{s}=\sum_{i \mathbf{q} \omega} \omega^{2}\left|\Phi_{-i}^{-}(\mathbf{q}) u^{i}(\mathbf{q})\right|^{2} \operatorname{Re} \int_{-\infty}^{0} d t \mathrm{e}^{t(\varepsilon-i \omega)}\left(T^{+}(\mathbf{q}), T^{-}(-\mathbf{q}, t)\right)
$$

The general conclusions about the frequency spectrum structure, the resonance line width, and the possibility of observing the resonance can be considered from the Green's function

$$
\begin{aligned}
G^{n}\left(\mathbf{q}, t-t_{1}\right)= & \theta\left(t-t_{1}\right) \mathrm{e}^{\left(t_{1}-t\right) \varepsilon}\left(T^{n}(\mathbf{q}, t), T^{-n}\left(-\mathbf{q}, t_{1}\right)\right)= \\
& =\int_{-\infty}^{\infty} \frac{d \omega}{2 \pi} \mathrm{e}^{i \omega\left(t_{1}-t\right)} G^{n}(\mathbf{q}, \omega)
\end{aligned}
$$

Composed as a chain for the Green function, coupled equations of motion can be formally solved in the Bohr approximation in interaction with scatterers in this way

$$
G^{n}(\mathbf{q}, \omega)=\frac{\left(T^{n}(\mathbf{q}), T^{-n}(-\mathbf{q})\right)}{M^{n}(\mathbf{q}, \omega)+\varepsilon+i\left(\Omega_{n}-\omega\right)} .
$$

The real part of the mass operator $\operatorname{Re} M^{n}(\mathbf{q}, \omega)=\Gamma^{n}(\mathbf{q}, \omega)$ holds for the width, and the imaginary one $\operatorname{Im} M^{n}(\mathbf{q}, \omega)$ for the resonance line shift due to the electron diffusion and the electron-lattice scattering. After neglecting the resonance frequency shift, we have

$$
Q_{s}=\beta \sum_{\mathbf{q} \omega} \omega^{2}\left|\Phi_{-i}^{-}(\mathbf{q}) u^{i}(\mathbf{q})\right|^{2} \frac{\left(s^{+}(\mathbf{q}), s^{-}(-\mathbf{q})\right) \Gamma^{ \pm}(\mathbf{q}, \omega)}{\left(\Gamma^{ \pm}(\mathbf{q}, \omega)\right)^{2}+\left(\omega_{s}-\omega\right)^{2}} .
$$

The line width up to the terms $\sim q^{2}$ and in the Bohr approximation in electron-lattice interaction appears as

$$
\Gamma(\mathbf{q}, \omega)=v(\mathbf{q}, \omega)+q^{\alpha} q^{\gamma} D_{\alpha, \gamma}^{ \pm}(\mathbf{q}, \omega),
$$


where $v(\mathbf{q}, \omega)$ is the known formula for a relaxation frequency of transverse electron spin; it defines the line width of the paramagnetic resonance [56]:

$$
\begin{gathered}
v(\mathbf{q}, \omega)=\frac{1}{\left(s^{+}(\mathbf{q}), s^{-}(-\mathbf{q})\right)} \times \\
\times \operatorname{Re} \int_{-\infty}^{0} d t_{1} \mathrm{e}^{(\varepsilon-i \omega) t_{1}}\left(\dot{s}_{(s m)}^{+}(\mathbf{q}), \dot{s}_{(s m)}^{-}\left(-\mathbf{q}, t_{1}\right)\right),
\end{gathered}
$$

and $D_{\alpha, \gamma}^{ \pm}(\mathbf{q}, \omega)$ is the diffusion tensor of transverse components of the spin magnetization

$$
\begin{gathered}
D_{\alpha, \gamma}^{ \pm}(\mathbf{q}, \omega)=\frac{1}{\left(s^{+}(\mathbf{q}), s^{-}(-\mathbf{q})\right)} \times \\
\times \operatorname{Re} \int_{-\infty}^{0} d t_{1} \mathrm{e}^{(\varepsilon-i \omega) t_{1}}\left(I_{s^{+}}^{\alpha}(\mathbf{q}), I_{s^{-}}^{\gamma}\left(-\mathbf{q}, t_{1}\right)\right) .
\end{gathered}
$$

The diffusion tensor components involved in the line width of the acoustic spin resonance (23), as can be noticed, are the Green functions $G^{n k}(\mathbf{q}, \omega)$ (for $\mathbf{q}=0$ ). By analogy with (21), the latter, in turns, can be represented with their damping and diffusion coefficients. Finally, the correlation functions $\left(s^{+}(\mathbf{q}), s^{-}(-\mathbf{q})\right)$ are proportional to the static paramagnetic susceptibility of electron [56]

$$
\chi_{s}(\mathbf{q}, 0)=\frac{\beta\left(g_{s} \mu_{0}\right)^{2}}{2}\left(s^{+}(\mathbf{q}), s^{-}(-\mathbf{q})\right) .
$$

Equations (14), (15) and the kinetic coefficients (16), (17) solve the problem of macroscopic description of the nonequilibrium spin subsystems in terms of average magnetization densities.

Let energy flux be stationary between the subsystems. We average the equations over time. As a result, the set of the Eqs. (14), (15) acquires the form

$$
\begin{gathered}
\delta \beta_{s m}(\mathbf{x}) L_{s(s m)}(\mathbf{x})+Q_{s}=0 \\
\delta \beta_{m s}(\mathbf{x}) L_{m(s m)}(\mathbf{x})+\delta \beta_{m p}(\mathbf{x}) L_{m(m p)}(\mathbf{x})=0 \\
L_{i(j k)}(\mathbf{x})=\int d \mathbf{x}^{\prime} \int_{-\infty}^{0} d t^{\prime} \mathrm{e}^{\varepsilon t^{\prime}}\left(\dot{H}_{i(j k)}(\mathbf{x}), \dot{H}_{i(j k)}\left(\mathbf{x}^{\prime}, t^{\prime}\right)\right),
\end{gathered}
$$

where $\delta \beta_{i k}=\beta_{i}-\beta_{k}$.

From (26) it follows that if the phonon subsystem is slightly unbalanced $\beta_{p} \sim \beta$ (the uniform case), the spinwave current in the magnetic insulator (the magnetization is unbalanced as $\delta S^{z} \sim \delta \beta_{m}$ ) depends resonantly on the frequency of the external field and is given by

$$
\delta S^{z} \approx \frac{\beta \tau_{m(m p)} \chi_{s}}{\chi_{m}} \sum_{\omega} \omega^{2}\left|\Phi_{-i}^{-} u^{i}\right|^{2} \frac{v_{s}(\omega)}{\left(v_{s}(\omega)\right)^{2}+\left(\omega_{s}-\omega\right)^{2}} .
$$

Here $\chi_{m}$ is the static susceptibility of the localized spins, $\tau_{m(m p)}^{-1}$ is the magnon-phonon relaxation frequency

$$
\tau_{m(m p)}^{-1}=\left(S^{z}, S^{z}\right)^{-1} \int_{-\infty}^{0} d t_{1} \mathrm{e}^{\varepsilon t_{1}}\left(\dot{S}_{(m p)}^{z}, \dot{S}_{(m p)}^{z}\left(t_{1}\right)\right) .
$$

Thus, the resonance energy absorption of a sound wave by conduction electrons in the metal causes the deviation of the magnon subsystem of magnetic insulator from the equilibrium state also in a resonant manner. In met$\mathrm{al} /$ magnetic insulator/metal structures spin Seebeck effect, observed by converting the injected spin-wave current from dielectric to the charge one by the inverse spin Hall effect [22], in the nonmagnetic metal (Pt) attached to the magnetic insulator behave in a resonant manner under studied above conditions.

\section{Conclusions}

In this paper, we have proposed and developed the new method of spin-wave current generation in the magnetic part of the metal/ferromagnetic insulator hybrid structures. It is based on resonance excitation of conduction electron spin subsystem by sound wave field followed by the momentum transition to the ferromagnetic insulator due to exchange interaction. We have developed the microscopic theory of spin transport under conduction electron spins subsystem resonance excitation and thermal perturbation of the magnetic subsystem conditions. We have demonstrated the proposed excitation method to create a nonequilibrium magnon accumulation at the interface and to lead to the spin-wave current resonance generation in the magnetic insulator. The explicit forms of the kinetic coefficients as correlation functions determining both diffusion and relaxation processes were found. In metal/magnetic insulator/metal structures under studied conditions spin Seebeck effect occure in a resonant manner.

\section{Acknowledgments}

The given work has been done as the part of the state task on the theme "Spin" 01201463330 (project 15-17-2-17) with the support of the Ministry of Education of the Russian Federation (Grant 14.Z50.31.0025), and RFBR (Grant 16-02-00044).

\section{Appendix}

The operators $T^{n}(\mathbf{q})$, in the definition of Green's function satisfy the equation of motion

$$
\begin{gathered}
\dot{T}^{n}(\mathbf{q})=(i \hbar)^{-1}\left[T^{n}(\mathbf{q}), H\right]= \\
=-i \Omega_{n} T^{n}(\mathbf{q})+i \mathbf{q}^{-k}(1 / m) T^{n k}(\mathbf{q})+\dot{T}_{(v)}^{n}(\mathbf{q}) .
\end{gathered}
$$


Here

$$
T^{n}(\mathbf{q}) \equiv \sum_{i} s_{i}^{\mu}\left\{p_{i}^{k}, \mathrm{e}^{i \mathbf{q} x_{i}}\right\}, \quad \dot{T}_{(v)}^{n}(\mathbf{q})=(i \hbar)^{-1}\left[T^{n}(\mathbf{q}), H_{(v)}\right],
$$

$\Omega_{n}$ are the precession frequencies of $T^{n}(\mathbf{q})$ in magnetic field, being resonance ones of acoustic resonance; $(1 / m) T^{n k}(\mathbf{q})$ describes a diffusive flow of inhomogeneous distribution, $T^{n}(\mathbf{q})$, a $\dot{T}_{(v)}^{n}(\mathbf{q})$ determines the rate of change of the values during interaction of the electrons with the lattice. Green's functions satisfy the chain of equations

$$
\begin{gathered}
\left(i\left(\Omega_{n}-\omega\right)+\varepsilon\right) G=I-G_{1}, \\
\left(i\left(\Omega_{n}-\omega\right)+\varepsilon\right) G_{1}=I_{1}+G_{2},
\end{gathered}
$$

where

$$
\begin{gathered}
I=\left(T^{n}(\mathbf{q}), T^{-n}(-\mathbf{q})\right), \\
I_{1}=\left(T^{n}(\mathbf{q}),-i \mathbf{q}^{k}(1 / m) T^{-n-k}(-\mathbf{q})+\dot{T}_{(v)}^{-n}(-\mathbf{q})\right), \\
G \equiv G^{n}(\omega), \\
G_{1}=\int_{-\infty}^{0} \mathrm{e}^{(\varepsilon-i \omega) t}\left(T^{n}(\mathbf{q}),-q^{k}(1 / m) T^{-n-k}(-\mathbf{q}, t)+\dot{T}_{(v)}^{-n}(-\mathbf{q}, t)\right), \\
G_{2}=\int_{-\infty}^{0} \mathrm{e}^{(\varepsilon-i \omega) t}\left(i q^{-k}(1 / m) T^{n k}(\mathbf{q})+\right. \\
\left.+\dot{T}_{(v)}^{n}(\mathbf{q}),-q^{k}(1 / m) T^{-n-k}(-\mathbf{q}, t)+\dot{T}_{(v)}^{-n}(-\mathbf{q}, t)\right) .
\end{gathered}
$$

With the help of Green's chain of equations the mass operator $M=G_{1} G^{-1}$ can be represented as

$$
\begin{gathered}
M=\frac{I_{1}+G_{2}}{I-G_{1}}=\frac{1}{I}\left(I_{1}+G_{2}+G_{1}^{2} G^{-1}\right), \\
\Gamma=\frac{1}{I} \operatorname{Re}\left(G_{2}+G_{1}^{2} G^{-1}\right) .
\end{gathered}
$$

1. K. Uchida, S. Takanashi, K. Harii J. Jeda, W. Koshibae, K. Ando, S. Maekawa, and E. Saitoh, Nature 455, 778 (2008).

2. C.M. Jaworski, J. Yang, S. Mack, D.D. Awschalom, J.P. Heremans, and R.C. Myers, Nat. Mater. 9, 898 (2010).

3. K. Uchida, J. Xiao, H. Adachi, J. Ohe, S. Takahashi, J. Ieda, T. Ota, Y. Kajiwara, H. Umezawa, H. Kawai, G.E.W. Bauer, S. Maekawa, and E. Saitoh, Nat. Mater. 9, 894 (2010).

4. Z. Ma, Solid State Commun. 150, 510 (2010).

5. Xuele Liu and X.C. Lie, Solid State Commun. 150, 471 (2010).

6. Shu-guang Cheng, Yanxia Xing, Oing-feng Sun, and X.C. Xie, Phys. Rev. B 78, 045302 (2008).

7. A. Dyrdal and J. Barnas, J. Phys.: Condens. Matter 24, 275302 (2012).
8. Laurent Gravier, Santiago Serrano-Guisan, Francois Reuse, and J.-Ph. Ansernet, Phys. Rev. B 73, 052410 (2006).

9. S.Y. Huang, W.G. Wang, S.F. Lee, J. Kwo, and C.L. Chien, Phys. Rev. Lett. 107, 216604 (2011).

10. G.E.W. Bauer, A.H. MacDonald, and S. Maekawa, Solid Staste Commun. 150, 459 (2010).

11. Gerrit E. Bauer, Eiji Saitoh, and Bart J. van Wees, Nat. Mater. 11, 391 (2012).

12. H. Adachi, J. Ohe, S. Takahashi, and S. Maekawa, Phys. Rev. B 83, 094410 (2011).

13. H. Adachi, K. Uchida, E. Saitoh, and S. Maekawa, Rep. Progr. Phys. 76, 036501 (2013).

14. J. Xiao, Gerrit E.W. Bauer, K. Uchida, E. Saitoh, and S. Maekawa, Phys. Rev. B 81, 214418 (2010).

15. K. Nakata, J. Stat. Mech: Theory and Experiment P05020, 1742 (2012).

16. K. Uchida, T. Nonaka, T. Ota, and E. Saitoh, Appl. Phys. Lett. 97, 262504 (2010).

17. Y. Kajiwara, K. Harri, S. Takahashi, J. Ohe, K. Uchida, M. Mizuguchi, H. Umezawa, H. Kawai, K. Ando, K. Takanashi, S. Maekawa, and E. Saitoh, Nature 464, 262 (2010).

18. S. Takahashi, E. Saitoh, and S. Maekawa, J. Phys.: Conf. Ser. 200, 062030 (2010).

19. F.L. Bakker, A. Schlachter, J.-P. Adam, and B.J. van Wees, Phys. Rev. Lett. 105, 136601 (2010).

20. S.O. Valenzuela and M. Tinkham, J. Appl. Phys. 101, 09B103 (2007).

21. Y.K. Kato, R.C. Myers, A.C. Gossard, and D.D. Awschalom, Science 306, 1910 (2004).

22. E. Saitoh, M. Ueda, H. Miyajima, and G. Tatara, Appl. Phys. Lett. 88, 182509 (2006).

23. T. Kimura, Y. Otani, T. Sato, S. Takahashi, and S. Maekawa, Phys. Rev. Lett. 98156601 (2007).

24. Koki Takanashi, Jpn. J. Appl. Phys. 49, 110001 (2010).

25. J. Sinova, D. Culcher, Q. Niu, N.A. Sinitsyn, T. Jungwirth, and A.H. MacDonald, Phys. Rev. Lett. 92, 126603 (2004).

26. K. Ando, S. Takahashi, J. Jeda, H. Kurebayashi, T. Trypiniotis, C.H.W. Bauer, S. Maekawa, and E. Saitoh, Nat. Mater. 10, 655 (2011).

27. T. Seki, Y. Hasegawa, S. Mitani, S. Takahashi, H. Imamura, S. Maekawa, J. Nitta, and K. Takanashi, Nat. Mater. 7, 125 (2008).

28. L. Vila, T. Kimura, and Y. Otani, Phys. Rev. Lett. 99, 226604 (2007).

29. A. Azevedo, L.H. Vilela Leго, R.L. Rodriguez-Suarez, A.B. Oliveira, and S.M. Rezende, J. Appl. Phys. 97, 10C715 (2005).

30. E.I. Rashba, Phys. Rev. B 62, R16267 (2000-II).

31. H.J. Zhu, M. Ramsteiner, H. Kostial, M. Wassermeier, H.-P. Schonherr, and K.H. Ploog, Phys. Rev. Lett. 87, 016601 (1996).

32. B.T. Jonker, G. Kioseoglou, A.T. Hanbicki, C.H. Li, and P.E. Thompson, Nature Phys. 3, 542 (2007).

33. X. Lou, C. Adelmann, M. Furis, S.A. Crooker, C.J. Palmstrom, and P.A. Crowell, Phys. Rev. Lett. 96, 176603 (2006). 
34. K. Uchida, H. Adachi, T. An, H. Nakayama, M. Toda, B. Hillebrands, S. Maekawa, and E. Saitoh, J. Appl. Phys. 111, 053903 (2012).

35. K. Uchida, T. Ota, H. Adachi, J. Xiao, T. Nonaka, Y. Kajawara, G.E. Bauer, S. Maekawa, and E. Saitoh, J. Appl. Phys. 111, 103903 (2012).

36. E.I. Rashba, Phys. Usp. 7, 823 (1965).

37. A.B. Roitsin, Phys. Usp. 14, 766 (1972).

38. V.P. Kalashnikov, Theor. Mathem. Physics 18, 76 (1974).

39. V.P. Kalashnikov and I.I. Lyapilin, Theor. Mathem. Physics 18, 194 (1974).

40. Y. Yafet, Solid State Phys. 96, 198 (1964).

41. A.I. Akhiezer, M.I. Kaganov, and G.Y. Liubarskii, Z. Eksp. Theor. Fiz. 32, 837 (1957).

42. I.I. Lyapilin, M.S. Okorokov, and V.V. Ustinov, Phys. Rev. B 91, 195309 (2015).

43. I.I. Lyapilin, J. Acoust. Soc. Am. 133, 1894 (2013).

44. K. Ucida, H. Adachi, T. An, T. Ota, M. Toda, B. Hillebrandes, S. Maekawa, and E. Saitoh, Nat. Mater. 10, 737 (2011).

45. A. Overhauser, Phys. Rev. 89, 689 (1953).
46. C.A. Al'tshuler, B.I. Kochelaev, and A.M. Leushin, Physics Uspekhi 4, 880 (1962).

47. N. Mikoshiba, Phys. Lett. 12, 289 (1964).

48. R. Kubo, M. Yokota, and S. Nakajima, J. Phys. Soc. Jpn 12, 1203 (1957).

49. J.M. Luttinger, Phys. Rev. 135, A1505 (1964).

50. L. Onsager, Phys. Rev. 37, 405 (1931).

51. H. Greene, J. Math. Phys. 2, 344 (1961).

52. D.N. Zubarev, Nonequilibrium Statistical Thermodynamics, Nauka, Moscow (1971) [in Russion].

53. D.N. Zubarev and V.P. Kalashnikov, Theor. Mathem. Phys. 1, 137 (1969).

54. R.M. White, Quantum Theory of Magnetism; McGraw-Hill, Inc., New York (1970).

55. S.V. Vonsovskii, Ferromagnetic Resonance, Fiz.-Mat. Lit., Moscow (1961).

56. H.M. Bikkin and V.P. Kalashnikov, Theor. Mathem. Physics 7, 79 (1971). 